\title{
The International Atomic Energy Agency global initiatives on nasopharyngeal cancer treatment
}

\author{
Ka-On Lam ${ }^{1}$, Anne W. M. Lee ${ }^{1}$, Wai-Tong $\mathrm{Ng}^{2}$, Kirsten I. Hopkins ${ }^{3}$ \\ ${ }^{1}$ Department of Clinical Oncology, LKS Faculty of Medicine, The University of Hong Kong, Hong Kong, China; ${ }^{2}$ Department of Clinical \\ Oncology, Pamela Youde Nethersole Eastern Hospital, Hong Kong, China; ${ }^{3}$ International Atomic Energy Agency, Vienna, Austria \\ Contributions: (I) Conception and design: KO Lam, AW Lee, WT Ng; (II) Administrative support: KO Lam, AW Lee; (III) Provision of study \\ materials or patients: KO Lam, KI Hopkins; (IV) Collection and assembly of data: KO Lam, KI Hopkins; (V) Data analysis and interpretation: All \\ authors; (VI) Manuscript writing: All authors; (VII) Final approval of manuscript: All authors. \\ Correspondence to: Anne W. M. Lee, MD. Clinical Professor, Department of Clinical Oncology, LKS Faculty of Medicine, The University of Hong \\ Kong, 102 Pokfulam Road, Hong Kong, China. Email: awmlee@hku.hk.
}

\begin{abstract}
Since its establishment in 1957, the International Atomic Energy Agency (IAEA) has striven to promote global access to reliable and affordable radiotherapy, with much of its latest focus being in developing countries. While nasopharyngeal cancer (NPC) is highly curable by radiotherapy with excellent outcomes in developed countries, the outcomes in low- and middle-income countries (LMICs) are disappointing. Although the reasons for this are complex and multifactorial, improving the accessibility and quality of radiotherapy in these countries is fundamental. With concerted effort from experts and advocates around the world, ongoing initiatives are supported by the IAEA to combat the challenges in LMICs.
\end{abstract}

Keywords: Nasopharyngeal cancer (NPC); International Atomic Energy Agency (IAEA); radiotherapy access; low-middle income countries

Submitted Jan 01, 2016. Accepted for publication Jan 28, 2016.

doi: $10.21037 /$ cco.2016.03.02

View this article at: http://dx.doi.org/10.21037/cco.2016.03.02

\section{The International Atomic Energy Agency (IAEA): together against cancer}

The IAEA was set up in 1957 as the world's center for cooperation in the nuclear field (1). It works with its Member States and multiple partners worldwide to promote the safe, secure and peaceful use of nuclear technologies. While the IAEA was initially established in response to the deep fears and great expectations arising from the discovery of nuclear energy, its programs that have brought the greatest benefit to developing countries include those related to the use of radiation in human health. The contribution of the IAEA in the treatment of cancer with radiotherapy can be dated back to 1958 when its Division of Life Sciences began to support the applications of radiation in medicine, dosimetry, the environment and radiobiology. In the past five decades, its works have been shifting from basic research in radiation biology to the design and provision of clinical radiation facilities, especially in low- and middle-income countries (LMICs). Since 1990, the main focus of the program has been health care solutions in developing countries ranging from the design of national strategies for cancer diagnosis and therapy to procurement of equipment and training of essential staff $(2,3)$. Through the Program of Action for Cancer Therapy (PACT), IAEA has conducted more than 70 impact missions in LMICs and mobilized more than $€ 30$ million to improve cancer diagnosis and treatment in the 10 years since its establishment. The imPACT Review, a cancer control assessment tool, provides an evaluation of a country's readiness to implement cancer control programs to meet the cancer crisis. The Advisory Group on Increasing Access to Radiotherapy Technology (AGaRT) and Virtual University for Cancer Control (VUCCnet) are remarkable programs under the PACT umbrella. The former brings together radiotherapy equipment suppliers and radiotherapy users from developing countries to ensure that radiotherapy 


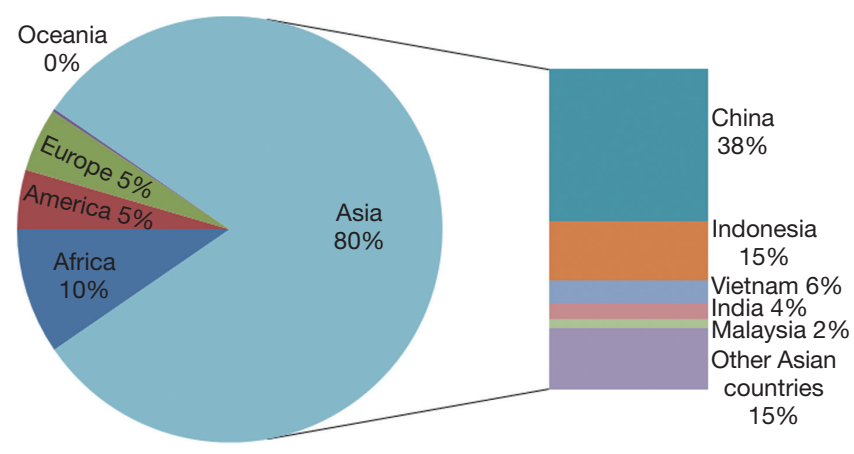

Figure 1 Epidemiological pattern of NPC: incidence in different continents with breakdown of the top five countries (Data from GLOBOCAN 2012). NPC, nasopharyngeal cancer.

technology is tailored to meet their service requirements. The latter program addresses the drastic shortage of accessible knowledge and quality training for cancer control by using e-learning and traditional teaching approaches to develop human resources in cancer control. Apart from these, the Technical Cooperation Program is uniquely positioned to support relevant and comprehensive cancer control proposals submitted by the Member States in 2-year planning cycles (4).

One example of the attempts by IAEA to improve treatment outcomes is a prospective randomized trial conducted to evaluate the addition of a brachytherapy boost in patients with advanced nasopharyngeal cancer (NPC) treated by standard chemoradiotherapy in participating centers from Egypt, Algeria, Morocco, Pakistan and Thailand (5). The study did not demonstrate a significant improvement in local failure-free survival by adding brachytherapy. However, it revealed the important observation that local control was poor in these centers, even in patients with $\mathrm{T} 1-2 \mathrm{~N}+$ disease (3-year local failurefree survival was $58 \%$ with brachytherapy $v s$. $52 \%$ without brachytherapy, $\mathrm{P}=0.34$ ).

\section{NPC: an ideal model for studying RT access}

NPC has a much skewed geographical and ethnic distribution. According to the epidemiological data from GLOBOCAN 2012 by the International Agency for Research on Cancer (IARC), around $80 \%$ of new cases occurred in Asia with another 10\% in Africa. Indeed the top five countries that had the highest number of new cases of NPC (China, Indonesia, Vietnam, India and Malaysia) were all Asian countries and together they accounted for nearly
$70 \%$ of the global burden (Figure 1) (6).

NPC is one of the most challenging cancers because of its highly malignant natural behavior and anatomical proximity to critical structures. Radiotherapy is the primary treatment modality; the best quality radiotherapy is needed to ensure accurate delivery of high-dose radiation to the whole tumor extent with maximum protection of normal structures. Chemotherapy is added for patients with advanced loco-regional disease, as this confers an absolute benefit of $6.3 \%$ in 5 -year overall survival (HR 0.79, $95 \%$ CI, 0.73-0.86, $\mathrm{P}<0.0001)$ (7). Surgery is mainly used for diagnosis and salvage of recurrent diseases.

In addition to the requirement for precise, conformal radiotherapy, the best quality imaging to delineate the tumor extent is crucial. Advancements in imaging techniques, in particular the increasing availability of MRI, have also contributed to the steady improvement in treatment results. A study on the evolution of treatment of NPC showed that 5 -year overall survival increased from around $70 \%$ in the 2 -dimensional radiotherapy era in the 1990 s to over $80 \%$ in the contemporary intensity-modulated radiotherapy era (8).

Unfortunately, there is wide variation in treatment outcomes for NPC and health care resources in different countries. Most of the countries with the highest number of new NPC cases were low-income or low-middle-income countries as defined by the World Bank $(6,9)$, and their outcomes remain disappointingly poor. Recent reports from a center in Yogyakarta of Indonesia showed an alarming median overall survival of only 21 months (10). Another retrospective study of patients treated with radical chemo/ radiotherapy in Iran showed similar poor results with 2-year overall survival of only $35 \%$ (11).

The reasons for the poor outcomes in the LMICs are multifactorial. Both the quantity and quality of radiotherapy services are key contributing factors. The association between socio-economic status and the availability of radiotherapy equipment is well-documented in LMICs (12); there is a wide variation in radiotherapy resources even within Europe. As shown in the ESTRO-HERO survey, the access to radiotherapy facilities amount to 7 to 8 - fold difference across Europe: the number of $\mathrm{MV}$ machines per million population ranged from 1.4 to 9.5 (median 5.3) (13).

In an attempt to demonstrate the correlation between the access to radiotherapy and treatment outcomes of NPC at a global level, we have extracted the epidemiologic data from GLOBOCAN and the radiotherapy resource data from the Directory of Radiotherapy Centers of the IAEA (IAEA-DIRAC). There is a wide variation in access to 


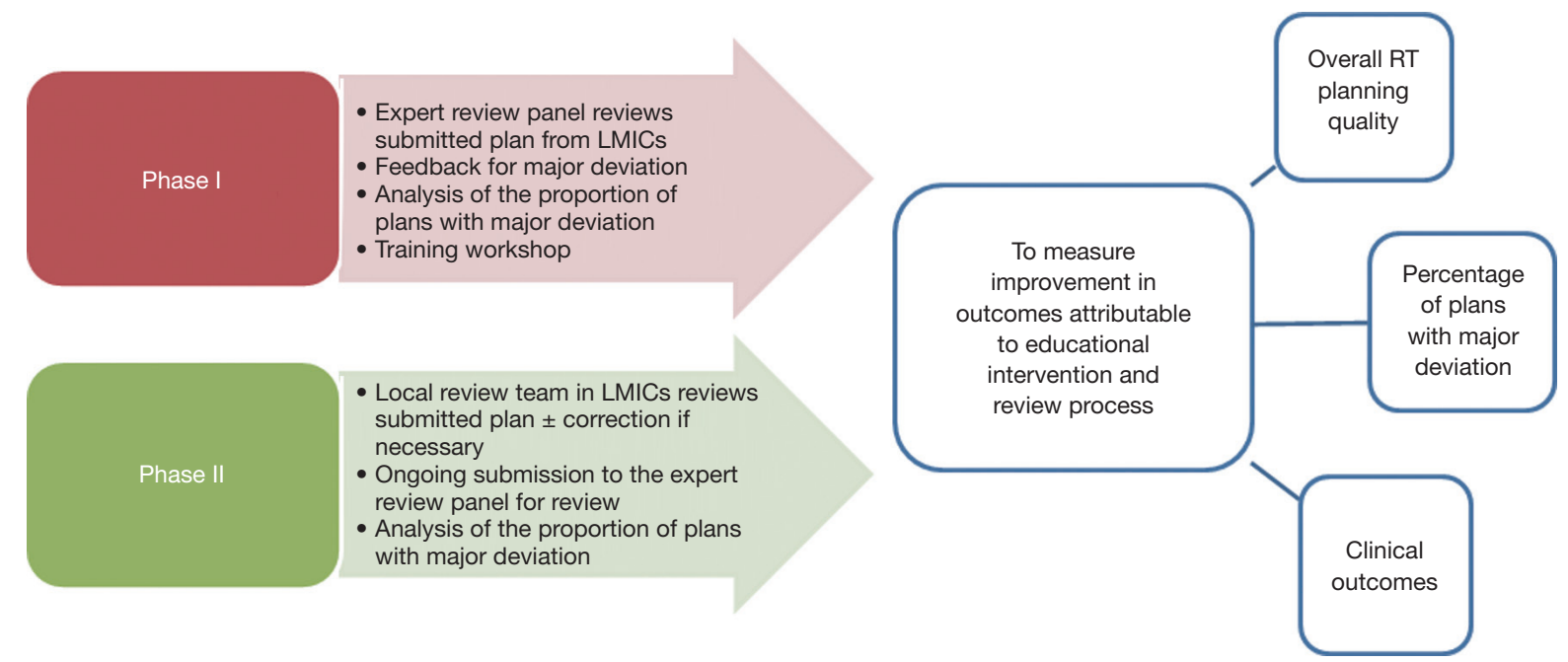

Figure 2 Schematic diagram of the current IAEA proposal in LMICs. IAEA, International Atomic Energy Agency; LMICs, low- and middle-income countries.

radiotherapy around the world: the number of radiotherapy equipment units ranges from 0.02-12.6 (median 1.6) per million populations. Using the [1-(age-standardized mortality rate/age-standardized incidence rate)] as the proxy relative survival, 112 countries were categorized into Poor, Median and Good Outcomes Groups. Our analyses showed significant correlation between treatment outcomes and the access to radiotherapy. Sadly only $18 \%$ of Asian countries and 5\% of African countries had Good Outcome as compared to $53 \%$ in Europe (14).

Besides the availability of radiotherapy equipment, access to good quality radiotherapy is equally crucial. Modelling of Quality Assurance in Radiotherapy (QART) in an EORTC head and neck cancer study has suggested clinical benefit from increasing QART and that this approach is costeffective (15). An analysis from a multi-centre international trial on other head and neck cancers has demonstrated that better protocol compliance is associated with significantly improved outcomes in comparison to cases with a major protocol violation: the absolute magnitude of improvement amounted to over $20 \%$ in 2-year loco-regional failure rate (46\% vs. $22 \%$ ) and overall survival (70\% vs. $50 \%)$ (16).

\section{Investing in cancer control in LMICs: no longer an option, but a responsibility}

Since good quality radiotherapy planning is a fundamental part of providing a good quality radiotherapy service, the IAEA has taken the pioneer role to launch a coordinated research project to improve the treatment outcomes of NPC patients in the LMICs. Firstly, a national survey will be conducted to gather updated demographic data and radiotherapy access in the targeted LMICs which have a relatively high incidence of NPC but poor treatment outcomes (Figure 2). Secondly, a cross-sectional study on the characteristics of NPC patients and detailed pattern of care in participating centers will be performed. Thirdly, a comparative study on quality of RT planning and clinical outcomes before and after an educational intervention and establishment of local quality control will be conducted. Participating centers will be required to submit radiotherapy datasets that contain clinical details, the radiotherapy plan and prescription for consecutive patients in two phases. The submissions will be reviewed by a review panel composed of international experts. The first phase (group A) will provide baseline information about the quality and practice in the participating centers; feedback will be given only if serious deviation is noted. Technical evaluation of the baseline plans will be summarized and an Educational Workshop will then be conducted 'to train the trainer' to improve their knowledge and skills in RT planning and to establish Independent Local Review Team. In the second study phase (group B), the radiotherapy plans should be reviewed first by the Local Review Team and corrections (if necessary) implemented. The intended plan will then be submitted to the expert panel for review and feedback will be given as quickly as possible to minimize deviation. To prove whether the review process and educational intervention translates 
into clinical benefits, the quality of radiotherapy planning and treatment outcomes will be compared between the two groups. Through this study, it is earnestly hoped that a robust practical model can be established and international collaboration can be enhanced to bring about a sustainable improvement in radiotherapy services in the LMICs.

\section{Conclusions}

One of the missions of IAEA is to harness the power of radiation for the greatest benefit in health care. A major aspect is to help LMICs to develop national strategies for cancer diagnosis and therapy, procure equipment and train essential staff. NPC is highly curable by good quality radiotherapy, but currently the outcomes remain poor in LMICs where this peculiar cancer is most prevalent. This project will be an ideal model for studying access to radiotherapy and improving quality control through educational intervention. It is earnestly hoped that concerted global efforts will improve equity of access to universal, high quality radiotherapy and hence improve outcomes for patients with NPC.

\section{Acknowledgements}

None.

\section{Footnote}

Conflicts of Interest: The authors have no conflicts of interest to declare.

\section{References}

1. Fisher D. History of the International Atomic Energy Agency: The first 40 years. Vienna, 1997. Available online: http://www-pub.iaea.org/mtcd/publications/pdf/pub1032 web.pdf

2. Enwerem-Bromson N, Abdel-Wahab M. Expanding global access to radiotherapy: the IAEA perspective. Lancet Oncol 2015;16:1151-2.

3. Rosenblatt E, Acuña $\mathrm{O}$, Abdel-Wahab M. The challenge of global radiation therapy: an IAEA perspective. Int J Radiat Oncol Biol Phys 2015;91:687-9.

4. International Atomic Energy Agency website. Available online: http://cancer.iaea.org/index.asp, accessed on 22 Nov 2015.

5. Rosenblatt E, Abdel-Wahab M, El-Gantiry M, et al.
Brachytherapy boost in loco-regionally advanced nasopharyngeal carcinoma: a prospective randomized trial of the International Atomic Energy Agency. Radiat Oncol 2014;9:67.

6. GLOBOCAN 2012. Available online: http://globocan.iarc. fr/Pages/online.aspx, accessed on 22 Nov 2015.

7. Blanchard P, Lee A, Marguet S, et al. Chemotherapy and radiotherapy in nasopharyngeal carcinoma: an update of the MAC-NPC meta-analysis. Lancet Oncol 2015;16:645-55.

8. Lee AW, Ng WT, Chan LL, et al. Evolution of treatment for nasopharyngeal cancer--success and setback in the intensity-modulated radiotherapy era. Radiother Oncol 2014;110:377-84.

9. The World Bank. Available online: http://data.worldbank. org/about/country-and-lending-groups\#Low_income, accessed on 22 Nov 2015.

10. Wildeman MA, Fles R, Herdini C, et al. Primary treatment results of Nasopharyngeal Carcinoma (NPC) in Yogyakarta, Indonesia. PLoS One 2013;8:e63706.

11. Khademi B, Mahmoodi J, Omidvari S, et al. Treatment results of nasopharyngeal carcinoma: a 15-year single institutional experience. J Egypt Natl Canc Inst 2006;18:147-55.

12. Datta NR, Samiei M, Bodis S. Radiation therapy infrastructure and human resources in low- and middleincome countries: present status and projections for 2020. Int J Radiat Oncol Biol Phys 2014;89:448-57.

13. Grau C, Defourny N, Malicki J, et al. Radiotherapy equipment and departments in the European countries: final results from the ESTRO-HERO survey. Radiother Oncol 2014;112:155-64.

14. Lam KO, Lee AW, Choi CW, et al. Global pattern of nasopharyngeal cancer: correlation of outcome with access to radiotherapy. Int J Radiat Oncol Biol Phys. In press.

15. Weber DC, Hurkmans CW, Melidis C, et al. Outcome impact and cost-effectiveness of quality assurance for radiotherapy planned for the EORTC 22071-24071 prospective study for head and neck cancer. Radiother Oncol 2014;111:393-9.

16. Peters LJ, O'Sullivan B, Giralt J, et al. Critical impact of radiotherapy protocol compliance and quality in the treatment of advanced head and neck cancer: results from TROG 02.02. J Clin Oncol 2010;28:2996-3001.

Cite this article as: Lam $\mathrm{KO}$, Lee $\mathrm{AW}, \mathrm{Ng}$ WT, Hopkins KI. The International Atomic Energy Agency global initiatives on nasopharyngeal cancer treatment. Chin Clin Oncol 2016;5(2):27. doi: 10.21037/cco.2016.03.02 